\title{
The impact of the America's Cup on Fremantle Hospital*
}

\author{
G. M. GALVIN \& G. A. JELINEK \\ Department of Emergency Medicine, Fremantle Hospital, Fremantle, Australia
}

\section{SUMMARY}

The first defence of the America's Cup outside of the USA took place off Fremantle, Western Australia, between October, 1986 and February, 1987. Fremantle Hospital,, the nearest hospital to the marinas, was involved in the planning for disasters and for the $\vec{\square}$ provision of clinical services to the syndicates involved and the projected crowds of tourists and spectators. The impact of this unique sporting event on the hospital was $\stackrel{D}{=}$ measured by collecting data from all patients whose presence in Fremantle was related. to the yachting. The America's Cup resulted in only 654 attendances and 31 admissions $\vec{\circ}$ over the 5 months of the event. The figures fell far short of those predicted. A number $9 f_{0}^{\circ}$ conclusions have been drawn.

\section{INTRODUCTION}

In 1983, the Australian $12 \mathrm{~m}$ yacht Australia II won the America's Cup and became th first non-American syndicate to do so in the 132-year history of the cup.

The America's Cup came to Western Australia and a challenge series was organized for late 1986 to early 1987 in the Indian Ocean off Fremantle. As this defence was the first to take place outside of the USA, it presented a number of problems to Western Australia.

Worldwide interest was expressed in challenging for the cup, media interest was high and responsible government departments forecast a boom in visitors at the time of the 9 defence. One forecast indicated that over the period of the Cup there would be one million extra visitors to Fremantle and its environs. At its peak, it was estimated there would be an additional 100-110 thousand people in Fremantle on any given day. N

*The subject matter of this paper was presented at the Second International Conference for Emergency
Medicine in Brisbane, October 1988.

Correspondence: $\operatorname{Dr}$ G.M. Galvin, Department of Emergency Medicine, Fremantle Hospital, PO Box 480 Fremantle, WA 6160, Australia. 
Challenge syndicates came from six countries, namely, USA, Canada, UK, France, Italy and New Zealand. Defence syndicates from Australia nominated six yachts, namely Australia III and IV, Kookaburra II and III, Steak 'n' Kidney, and South Australia. The marinas were all located around the Fremantle Boat Harbour and crews accommodated in Fremantle.

The City of Fremantle is said to be one of the best preserved areas of late Victorian and Edwardian architecture in the world. The city lies at the apex of a triangle and is bounded by the ocean and the mouth of the Swan River. The access roads are poor and the city is prone to traffic congestion. Fremantle Hospital was then a 363 bed teaching hospital within the University of Western Australia. The hospital is located $0.7 \mathrm{~km}$ from the marinas and dominates the skyline at that point. A full emergency service is provided and the Emergency Department has some 82000 occasions of service per year. It was expected that Fremantle Hospital would provide medical services for many visitors and play a leading role in disaster management.

\section{PLANNING}

When planning began for the America's Cup period, it was felt vital to get everything right because, if history repeated itself, Fremantle would host the event every 4 years for the next century. Early meetings between the various emergency services indicated an enormous potential for disaster. There would be huge crowds, traffic flow would be disrupted and, on the water, there would be a mix of boats from ocean liners to small run-abouts. The level of boat ownership in Perth is high and congestion was expected both to and from, as well as on the racing course. A preview of the event was provided when the QE2 liner visited Fremantle in 1984. Some 40000 spectators flocked to Fremantle and the city ground to a halt. Road movement became impossible.

Planning within the hospital was directed at disaster planning and provision of clinical services.

\section{Disaster Planning}

The external agencies involved in disaster planning were police, fire, ambulance, health department, department of harbours and marine, Fremantle Port Authority and state emergency services as well as the Race Committee from the Royal Perth Yacht Club. The hospital protocols were revised and coordination meetings held with the other agencies. Specific landing points for water casualties were made. Rotary wing evacuation from the course to the hospital was considered but not used. It was felt that the skies over the course would be heavily congested with other helicopters and airships, and in any event, water transport to the nominated landing areas was rapid, with only a short distance remaining to the hospital by road.

\section{Clinical Service Planning}

The America's Cup was considered to be a unique sporting event. Most major 
international events are concluded over 2 weeks. This was an event running over $5 \stackrel{\overline{0}}{\mathrm{\Phi}}$ months. It was felt that additional medical and nursing staff would be needed. Funds to $\frac{0}{2}$ employ an additional four medical staff were provided. Annual leave for medical and $\underset{\varrho}{\alpha}$ nursing staff was greatly restricted between December 1986 and February 1987. On the medical side this had the effect of adding an additional 8 staff members. These were all $\stackrel{\overline{\vec{S}}}{\stackrel{5}{9}}$ employed in the Emergency Department.

Additional consulting and treatment rooms were provided by rehabilitating old areas $\frac{\bar{\sigma}}{\bar{N}}$ that had been 'mothballed' some 5 years previously.

It was decided to carry out a prospective study on the effect of the Cup on Fremantle Hospital. Time limits were selected as the day of the first elimination race (5th October, $\rightarrow$ 1986) and the day after the final race (ultimately 5th February, 1987). Every patient $\stackrel{\circ}{\circ}$ attending the Emergency Department between those dates was asked the following $\vec{\omega}$ question:

'Is your presence in Fremantle today in any way related to the America's Cup?'

Those who answered in the affirmative had statistical information collected.

\section{RESULTS}

There were 540 individual patients who required 654 occasions of service. These $\stackrel{\mathbb{Q}}{-}$ services were provided in greatest proportion to Australians (211) and citizens of the $\overrightarrow{0}$ USA (199). The 654 visits generated 123 pathology requests, 144 sets of radiographs and 328 prescriptions were dispensed. Only 31 of the 540 patients were admitted for if patient care.

\section{DISCUSSION}

The crowds predicted to flock to Fremantle never came, much as happened in Los Angeles in 1984. The results show that over 5 months, only 654 occasions of service and 31 admissions could be attributed to the Cup. Compared to 82000 per annum this is not even a discernible blip on the attendance graph.

There were no disasters and none of the plans had to be implemented.

From the medical point of view, it was only a 'Rio-in-Freo' night on New Year's Eve that caused extra work. This resulted from big crowds being attracted to a City Mardi- 윽 Gras and little to do but drink alcohol. The cases seen over that night reflected mass $\frac{D}{O}$ intoxication. To cover similar events on the last night of the cup, an additional seven resident medical officers were placed on call but the crowd was well behaved and their of services were not required.

The additional rooms were never used but have now been used for another purpose. N

The extra medical staff were not required and the Emergency Department was, in $\mathcal{O}^{\circ}$ fact, over-staffed. When the staff began to take their leave over a compressed period it $\stackrel{\bullet}{\frac{C}{D}}$ created a staff shortage. 


\section{CONCLUSIONS}

The medical impact of the America's Cup on Fremantle Hospital was trivial; accurately predicting tourist flow to a unique sporting event is difficult; Fremantle Hospital planned well for the America's Cup but did not need to implement any plans; overstaffing, by restricting leave at times of potential crisis created significant staff shortages remote from the event; given the same set of circumstances, in the future, planning would be along similar lines. A hospital that fails to plan for major events with significant disaster potential may find it is overwhelmed. 